\title{
Building on bispecifics
}

Bispecific molecules that harness immune cells to recognize and eliminate cancer cells are emerging as a promising anticancer drug class. Two new molecules - a bispecific antibody and a bispecific tumourtargeted co-stimulatory agonist - that have enhanced effects in combination with other immunotherapies have recently been reported in Science Translational Medicine.

In the first paper, Crawford and colleagues at Regeneron describe the design of a CD3-engaging bispecific antibody that targets the glycoprotein mucin 16 (MUC16), which is overexpressed in ovarian cancer. Patients with ovarian cancer with $\mathrm{CD}^{+} \mathrm{T}$ cell infiltration have better outcomes, so the authors were interested in designing an antibody that could activate these $T$ cell infiltrates in the tumour.

Because bispecific antibodies induce a potent response, the choice of target is important to maximize specificity and limit toxicity. "Identifying targets against solid tumours has proven more challenging as the antigens are often expressed on certain normal tissues," says Crawford. "I think the choice comes down to: Is it overexpressed in the tumour? Does it have limited expression on normal tissues? Are the normal tissues that express this antigen accessible to antibodies?"

On the basis of these requirements, MUC16 seemed like a good candidate. The authors generated a fully human bispecific antibody (REGN4018) using the light chain from an anti-MUC16 antibody and an effector-minimized constant fragment $(\mathrm{Fc})$ to minimize the immunogenicity and limit anti-drug antibody responses.

In vivo, REGN4018 reduced tumour burden in immunedeficient mice transplanted with OVCAR- 3 cells and peripheral blood mononuclear cells. The authors also developed a syngeneic model to test REGN4018 in mice genetically engineered to express MUC16 in normal tissues, including the ovary, stomach and trachea and with a full immune system. REGN4018 significantly inhibited the growth of subcutaneously implanted xenografts compared with a CD3-binding control antibody.

Finally, the authors tested whether blockade of the PD-1 pathway can enhance the antitumour effects of REGN4018. The combination of REGN4018 and an anti-PD-1 antibody led to complete tumour clearance in four of the nine treated mice.

In the second paper, Umaña and colleagues describe the design of a next-generation agonist of the co-stimulatory receptor 4-1BB (also known as CD137). Other agonists of $4-1 \mathrm{BB}$, such as the antibody urelumab, have previously been tested in patients with solid tumours but caused dose-dependent hepatitis because 4-1BB crosslinks with $\mathrm{Fc} \gamma \mathrm{R}$-expressing cells in the liver.

To achieve potent $4-1 \mathrm{BB}$ stimulation at the tumour site without systemic $\mathrm{Fc} \gamma$ receptor $(\mathrm{Fc} \gamma \mathrm{R})$ crosslinking, Umaña and colleagues generated an antibody-shaped fusion protein that contains a trimeric 4-1BB ligand (4-1BBL) that binds $4-1 \mathrm{BB}$, a monovalent antigen-binding fragment (Fab) that binds a tumour antigen, and a mutated $\mathrm{Fc}$ region that is unable to bind $\mathrm{Fc} \gamma \mathrm{R}$ but can bind the neonatal Fc receptor ( $F c R n)$, which increases the potency and half-life of the drug. The authors generated two versions of the agonist by changing the Fab. One of them targeted CD19 (CD19-4-1BBL), which is expressed on malignant $B$ cells, and the other targeted fibroblast activation protein (FAP; FAP-4-1BBL), which is expressed in the tumour stroma.

The authors assessed the ability of FAP-4-1BBL to improve the functionality of tumour-infiltrating $\mathrm{T}$ cells in patient-derived tumour samples of FAP-expressing epithelial ovarian cancer, melanoma and lung adenocarcinoma. Stimulation with agonistic anti-CD3 and FAP-4-1BBL led to an increase

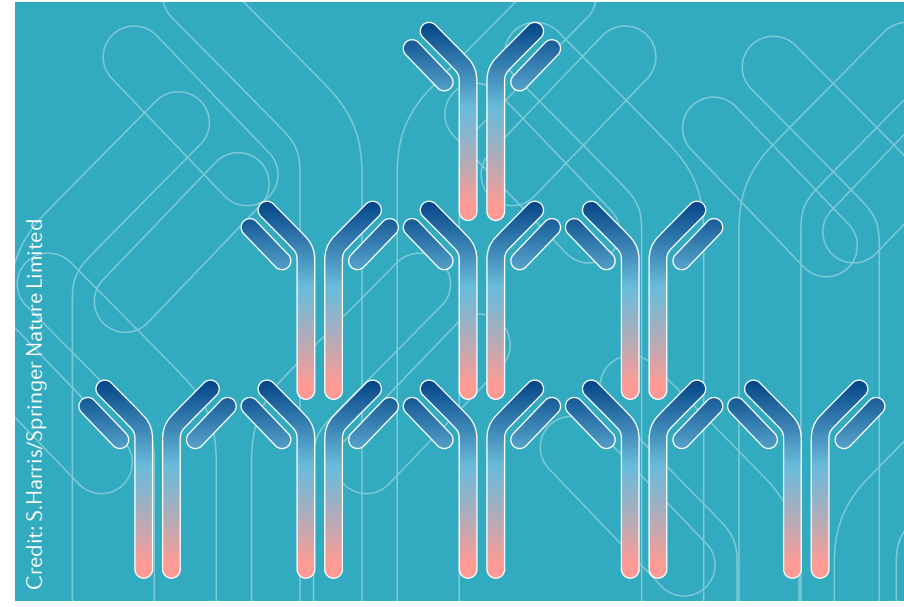

of several inflammatory cytokines in the supernatant in all samples.

In vivo, treatment of immunodeficient mice engrafted with human stem cells and bearing subcutaneous gastric cancer cell xenografts co-injected with mouse $\mathrm{NIH} / 3 \mathrm{~T} 3$ fibroblasts (to mimic FAP expression in patient samples) with a combination of FAP-4-1BBL and a carcinoembryonic antigen-targeted T cell bispecific antibody (CEA-TCB) inhibited tumour growth.

In addition, treatment of fully immunocompetent mice with a bispecific mouse surrogate antibody (muFAP-4-1BB) resulted in tumour remission when combined with muCEA-TCB or muPD-L1.

The authors conducted similar experiments with CD19-4-1BBL in combination with CD20-TCB

Two new molecules a bispecific antibody and a bispecific tumourtargeted co-stimulatory agonist - that have enhanced effects in combination with other immunotherapies have recently been reported
(CD20 is also expressed on B cells) in mice bearing an aggressive human lymphoma cell line. Treatment improved tumour control and increased $\mathrm{T}$ cell infiltration.

In summary, these two approaches successfully stimulate $T$ cells and improve survival in multiple mouse models, indicating the potential for more effective combinations in cancer immunotherapy for solid tumours.

M. Teresa Villanueva

ORIGINAL ARTICLES Crawford, A. et al. A muci 16 bispecific $T$ cell-engaging antibody for the treatment of ovarian cancer. Sci. Transl Med. 11 , eaau7534 (2019) |Claus, C. et al. Tumor-targeted 4-1BB agonists for combination with T cell bispecific antibodies as off-the-shelf therapy. Sci. Transl Med. 11, eaav5989 (2019) FURTHER READING Labrijn, A. F. et al. Bispecific antibodies: a mechanistic review of the pipeline. Nat. Rev. Drug Discov. https://doi.org/10.1038/ s41573-019-0028-1 (2019) | Mayes, P. A. et al. The promise and challenges of immune agonist antibody development in cancer. Nat. Rev. Drug Discov. 17, 509-527 (2018) 